\title{
Novel Performance Index and Multi-layered Information Structure for Monitoring Quasi-static Voltage Problems
}

\author{
Le Xie, Student Member, IEEE, Jovan Ilić, Member, IEEE, and Marija D. Ilić, Fellow, IEEE
}

\begin{abstract}
In this paper we propose a novel performance index and a multi-layered information structure for monitoring potential quasi-static voltage problems in electrical power systems. The proposed performance index is based on the sensitivity of the smallest singular value of the power flow Jacobian matrix with respect to the corresponding system load level. This performance index is computed at different levels of system aggregation/decomposition, which we define as a "multilayered information structure". This information structure requires little or no information exchange among decomposed subsystems. Moreover, it can be seamlessly implemented in today's horizontally decomposed interconnections. Two multicontrol-area systems are used to illustrate the proposed performance index and information structure.
\end{abstract}

Index Terms-matrix decomposition, power system monitoring, singular value decomposition.

\section{INTRODUCTION}

$\mathrm{T}$ HIS paper is motivated by the need for a systematic framework to monitor and control stressed complex power system. As new technical, regulatory and economic drivers emerge in the electric power industry, no such comprehensive framework has been implemented to meet prespecified performance benchmarks over broad ranges of operating conditions. During abnormal conditions, like the August 2003 blackout, problems are two-fold: (a) for individual controller's logic design, there is a lack of adaptiveness over a broad range of operating conditions; and, (b) for system-wide operation, there is a lack of situational awareness of system's deviations outside of normal ranges [1].

The objective of this paper is to address the problem (b) above in the context of assessing quasi-static voltage stability.

This work was supported in part by the U.S. National Science Foundation ITR Project, and, in part, by the U.S. Department of Energy, National Energy Technology Laboratory, under Research and Development Solutions, LLC contract number DE-AM26-04NT41817.305.01.21.002.The authors greatly appreciate this financial help.

Le Xie and Jovan Ilić are with the Department of Electrical and Computer Engineering, Carnegie Mellon University, Pittsburgh, PA 15213 (e-mail: 1x@ece.cmu.edu, jilic@ece.cmu.edu).

Marija D. Ilić is a Professor in the Department of Electrical and Computer Engineering and Department of Engineering and Public Policy, Carnegie Mellon University, Pittsburgh, PA, 15213 (email: milic@ece.cmu.edu).
The problem (a) has been studied elsewhere, for example local feedback design of individual controllers is addressed in [2] and adaptive voltage control schemes in [3]. These papers show that it is generally not sufficient to entirely rely on local voltage control logic, regardless of how advanced the control logic is. In other words, to make system-wide behavior more adaptive, online information is needed to detect when a system is outside of normal operating ranges. Typical information needed about a system is either the minimum singular value of the system Jacobian [3, 4], or the direct and quadrature axes components of the generator armature current [2].

Both problems (a) and (b) effectively lead to the same key question concerning the minimum information exchange and system decomposition (information structure) for identifying abnormal system conditions, voltage problems, in particular. Implied in this question is the need for a structured approach to defining the information communication which guarantees adequate performance of voltage controllers over a wide range of operating conditions. Because of the communication cost constraints in terms of data gathering and processing, and the administrative boundary barriers, it is most desirable to limit communication, or be decentralized as much as possible.

The main contribution of this work is the potential of communicating quasi-static information for assessing voltage and reactive power status ${ }^{1}$ in a decentralized way. To explain the type of online problems which could be controlled by using only quasi-static methods described in this paper, we consider the system changes which gradually lead to degradation of relevant performance of the system. Such changes include (1) real and/or reactive power loading change and (2) loss of controllability caused by the saturation of key voltage controllers. For example, saturation of the field voltage amplitude of an Automatic Voltage Regulator AVR), results in a voltage controlled generator behaving like load whose voltage varies as a result of system changes, i.e., it is no longer directly controllable. Similarly, Static Var Compensator (SVC), shunt capacitor and/or On-Load Tap-

\footnotetext{
${ }^{1}$ Truly dynamic problems are unlikely to rely on control logic beyond local. The methods described in this paper and elsewhere require information and computation beyond what is doable before the system enters dynamic voltage collapse regime.
} 
Changing Transformers (OLTC) ${ }^{2}$ voltage control limits can be reached.

The basic idea of this work is that it could be possible to detect gradual voltage degradation due to loss of some of voltage controllers prior to the ultimate voltage collapse at early stages of major blackouts. If necessary communication and adaptive control logic exists, the remaining controllers could be more effective and ultimate dynamic problems could often be avoided.

The remainder of this paper is organized as follows. In the next section the mathematical model underlying the normal power system condition is revisited. Currently used performance index (PI) for monitoring quasi-static voltage problem is explained and its limitations discussed. In Section III, the proposed PI and information structure are introduced. The multi-layered information structure specifies the method of system aggregation/decomposition which is a combination of two decomposition methods: overlapping decomposition and epsilon decomposition. The first method is based on the system decomposition into administrative control areas. The second method uses the so called " $\boldsymbol{\varepsilon}$-decomposition" method to decompose an interconnection into subsystems based on the weak coupling. In Section IV, the proposed PI and information structure are illustrated in two systems. Finally in Section V, conclusions are given and future work is suggested.

\section{BACKGROUND}

Large-scale power interconnections are one of the most complex man-made systems. Voltage stability is concerned with the ability of a power system to maintain acceptable voltage levels at all buses in the system [5], [6]. In order to understand the temporal and spatial interdependencies in a large-scale power system, it is necessary to understand the basic models and assumptions underlying the operation during normal conditions. In [1], a structure-based general model containing a set of coupled differential and algebraic equations (DAE) was presented to describe the current hierarchical control scheme. Without loss of generality, we consider an interconnected power system shown in Fig. 1. This represents two interconnected administrative regions. The loads are assumed not to have significant inertia, thus they are modeled as static sinks of pre-specified real and reactive power. We assume that there are automatic voltage regulators and governor-turbine-generators controlling the generator dynamics. A generalized form of a closed-loop controlled generator can be modeled as:

$\dot{x}_{L C, j}^{P}=f_{L C, j}^{P}\left(x_{L C, j}^{P}, y_{L C, j}^{P}, y_{L C, j}^{P, r e f}, p_{L C, j}^{P}\right)$

where $x_{L C, j}^{p}$ are the state variables defining the system dynamics, $y_{L C, j}^{p}$ are the local coupling variables through which local dynamics of generator $\mathrm{j}$ interact with the rest of

\footnotetext{
2 In power flow calculations this is sometimes referred to as a PV bus becoming a PQ bus.
}

the system, $y_{L C, j}^{P, r e f}$ are the nominal set points for the coupling variables, and $p_{L C, j}^{p}$ are the parameters of a generator $\mathrm{j}$.

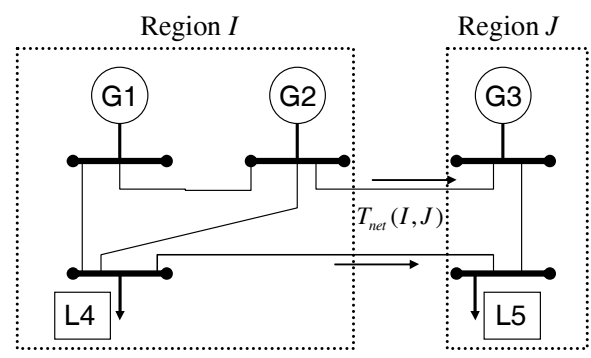

Fig. 1. One line diagram for interconnected 5-bus system.

The balance of real and reactive power at each instant of time is the network constraint. In terms of nodal equations, these constraints require the complex-valued power into the network $\hat{S}^{N}$ to be equal to the complex valued power $\hat{S}$ injected into each node.

$\hat{S}^{N}=\operatorname{diag}(\hat{V}) \hat{Y}_{\text {bus }}^{*}\left(\hat{V}^{*}\right)$

where $\hat{V}$ is the unknown complex node voltage vector, $\hat{Y}_{b u s}$ is the network nodal admittance matrix.

Combining the closed-loop dynamics of all generators inside the control area (1) with the network flow constraints (2), generally results in a coupled set of nonlinear DAEs of the form:

$$
\begin{aligned}
& \dot{x}=f\left(x, y, y^{r e f}, p, d\right), \quad x(0)=x_{0} \\
& 0=g\left(x, y, y^{r e f}, p, d\right)
\end{aligned}
$$

where $x$ are state variables defining the system dynamics of all system components and $y$ are coupling variables in the transmission lines. $p$ represent the system parameters such as forecast demand, damping of power plants, etc. The system disturbances are represented by vector $d$.

This model is a generalized description of the dynamics of an interconnected power system. It is of very high order for a typical control area or region. Because it is difficult to formalize control design for dynamic systems characterized as DAE models, current control designs first simplify the DAE model to a set of nonlinear ordinary differential equations (ODEs) [7]. One of the main assumptions for this simplification is the nonsingularity of matrix $J_{2}$, where

$J_{2}=\left[\begin{array}{ll}\partial P_{L} / \partial \theta_{L} & \partial P_{L} / \partial V_{L} \\ \partial Q_{L} / \partial \theta_{L} & \partial Q_{L} / \partial V_{L}\end{array}\right]$

$P_{L}$ and $Q_{L}$ represent the real and reactive power absorbed at load buses. $\theta_{L}$ and $V_{L}$ refer to the phase angle and voltage magnitude at load buses.

A nonsingular matrix $J_{2}$ reflects that, having specified real and reactive power load demand, one could compute the voltage magnitudes and angles at load buses. A nonsingular matrix $J_{2}$ is a necessary condition for the DAE model to be converted to ODE model, under which current hierarchical control is designed. Further more, the resulting ODE model of the form (3) is obtained by solving (4) for $y$ in terms of $x$, and (3) takes on the form of ODE. System $S$ is quasi-static 
when $\dot{x} \equiv 0$. It is known that under some modeling assumptions, the function $f\left(x, y, y^{r e f}, p, d\right)$ has the same form analogous to the complex power flow solutions [7].

The sensitivity of the quasi-static power flow solution with respect to changes in system state is defined as Jacobian matrix

$$
J=\frac{\partial f}{\partial x}=\left[\begin{array}{ll}
\partial P / \partial \theta & \partial P / \partial V \\
\partial Q / \partial \theta & \partial Q / \partial V
\end{array}\right]
$$

where $V$ is voltage magnitude vector whose voltage is not directly controlled (loads, in particular). $\theta$ is the vector of voltage phase angle of all but slack bus. There exists rich literature concerning the role and properties of power flow Jacobian matrix $J$ in relation to the possible potential quasistatic voltage problems. In practice, $J$ and $J 2$ can be used interchangeably to analyze the quasi-static voltage stability [1]. This is the starting model for the analysis in this paper.

There are two approaches in monitoring potential voltage problems: quasi-static (steady-state) approach and dynamic approach. While the dynamic approach takes the ODE model described above into computer-aided simulation, the quasistatic approach analyzes the proximity to voltage instability. Because of the time-consuming constraints and massive amount of data required for the dynamic analysis, the quasistatic voltage analysis is usually adopted in the current industry practice. A number of voltage stability indices were proposed in [8]. The smallest singular value of the power flow Jacobian matrix is widely used as a practical tool to determine the "distance" to quasi-static voltage collapse. The closeness of such value to zero can indicate the distance from the quasistatic voltage limit. This analysis still requires measurements from the entire system. However, due to the industry deregulation, it is necessary to implement a decentralized performance index to monitor the quasi-static voltage problem.

\section{Proposed Performance IndeX AND InFormation STRUCTURE}

\section{A. Proposed Performance Index (PI)}

Based on previous discussion, we propose a novel PI which can be defined as following:

Let $\sigma_{m}$ be the smallest singular value of matrix $J_{m}$.

Let $P_{0}=\sum_{i} P_{G}(i)$, where $P_{G}(i)$ is the net input of real power from generator $i$ into the system.

Let $Q_{0}=\sum_{i} Q_{G}(i)$, where $Q_{G}(i)$ is the net input of reactive power from generator $i$ into the network.

Let $S_{0}=\sqrt{P_{0}^{2}+Q_{0}^{2}}$, where $S_{0}$ represents the overall load level of the whole interconnection. Define PI as

$P I=\frac{\partial \sigma_{m}}{\partial S_{0}}$

If $P I<T$, where $T$ is the pre-specified threshold for the quasi-stationary voltage stability, the network is under normal operating condition. Otherwise if $P I \geq T$, the system is outside normal operating conditions. $\mathrm{T}$ is system dependent obtained through offline studies [9]. This proposed PI can be applied to decomposed sub groups (i.e. $J_{m}$ ), with little or no information exchange among the sub groups. In the next subsection, two network decomposition methods are explained.

\section{B. Possible Decomposition Methods}

\section{1) Overlapping Decomposition}

The existing power system interconnection is horizontally structured into a number of control areas. These control areas form the administrative boundaries. Each control area monitors and controls the power network within its boundary, while the neighboring control areas are interconnected via tie-lines. The administrative boundaries make it difficult for a control area to collect information outside its boundaries. Overlapping decomposition is based on the concept that each control area collects most of information (bus voltage magnitude, phase angle, real/reactive power flow) within its boundaries and exchanges with other areas as little information as possible. The information that is exchanged among control areas is the tie-line bus voltage magnitude and phase angle. The algorithm can be described as follows:

Let

$A=\{$ set of areas $\}=\left\{a_{1}, a_{2}, \ldots, a_{M}\right\}$, where $M$ is the total number of administrative areas;

$B=\{$ set of buses $\}=\left\{b_{1}, b_{2}, \ldots, b_{N}\right\}$, where $N$ is the total number of buses (nodes);

$A_{i}=\left\{\right.$ set of buses in area $\left.a_{i}\right\}$;

$\left\{A_{1}, A_{2}, \ldots, A_{M}\right\}$ is a disjoint partition of $B$, i.e., $A_{i} \cap A_{j}=\phi$ if $i \neq j ; \bigcup_{i=1}^{M} A_{i}=B$;

$T_{i}=\{$ set of buses that are directly connected to area $i$ but outside of area $i\}$;

The system Jacobian matrix model can be partitioned as

$\left[\begin{array}{l}\Delta S_{1} \\ \Delta S_{2} \\ \vdots \\ \Delta S_{M}\end{array}\right]=\left[\begin{array}{cccc}\partial \Delta S_{1} / \partial \Delta t_{1} & \partial \Delta S_{1} / \partial \Delta t_{2} & \cdots & \partial \Delta S_{1} / \partial \Delta t_{M} \\ \partial \Delta S_{2} / \partial \Delta t_{1} & \partial \Delta S_{2} / \partial \Delta t_{2} & \cdots & \partial \Delta S_{2} / \partial \Delta t_{M} \\ \vdots & \vdots & \vdots & \\ \partial \Delta S_{M} / \partial \Delta t_{1} & \partial \Delta S_{M} / \partial \Delta t_{2} & \cdots & \partial \Delta S_{M} / \partial \Delta t_{M}\end{array}\right]\left[\begin{array}{c}\Delta t_{1} \\ \Delta t_{2} \\ \vdots \\ \Delta t_{M}\end{array}\right]$

Here, $\Delta S_{i}$ refers to $\Delta P_{i}$ and $\Delta Q_{i}, \Delta t_{i}$ refers to $\Delta \theta_{i}$ and $\Delta V_{i}$.

The overlapping decomposition partitions the entire system model into $M$ subsystems. Tie-line buses are overlapped in neighboring sub-groups.

$$
\Delta S_{i}=\left[\begin{array}{cc}
\partial \Delta S_{i} / \partial \Delta t_{A i} & \partial \Delta S_{i} / \partial \Delta t_{T_{i}} \\
\partial S_{T_{i}} / \partial \Delta t_{A i} & \partial \Delta S_{T_{i}} / \partial \Delta t_{T_{i}}
\end{array}\right]\left[\begin{array}{c}
\Delta t_{A i} \\
\Delta t_{T_{i}}
\end{array}\right]
$$

where $i=1,2, \ldots, M . \Delta t_{A i}$ stands for the $\Delta \theta_{i}, \Delta V_{i}$ for buses in set $A_{i}$, and $\Delta t_{T i}$ stands for the $\Delta \theta_{i}, \Delta V_{i}$ for buses in set $T_{i}$.

In words, the overlapping decomposition takes buses within an area and buses directly connected to this area to form the sub-system's block Jacobian matrix.

Centralized power flow Jacobian is composed of four blocks (6). Under the assumption that the real-reactive power $(P-Q)$ decoupling holds, $\partial P / \partial \theta$ and $\partial Q / \partial V$ can be individually partitioned using the overlapping decomposition. ${ }^{3}$ Equation

\footnotetext{
${ }^{3}$ In principle, the information structures and performance index introduced in this paper can be applied without requiring the $P-Q$ decoupling. However, some
} 
(10) is a mathematical representation of overlapping decomposition for a two-area $J_{Q V}$ submatrix. Subscripts $a$ and $b$ correspond to nodes inside control area $\mathrm{a}$ and $\mathrm{b}$, respectively. Subscript $T$ represents the nodes which are connected by tie-lines.

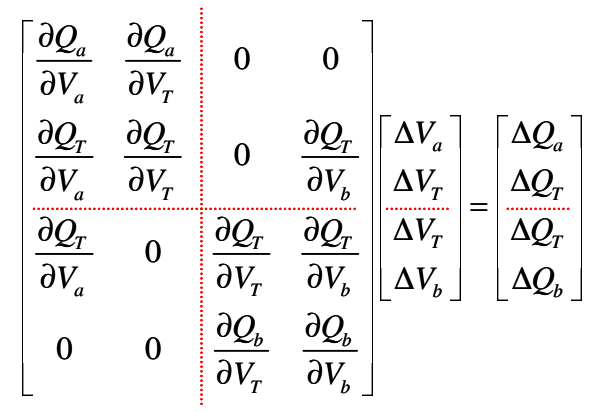

\section{2) Epsilon (E) Decomposition}

$\varepsilon$-decomposition method [10] is a clustering algorithm that decomposes a large interconnected system into several disjoint sub-groups. Algebraically, for any given matrix $A$, all the entries that are less than the given threshold $\varepsilon$ will be approximated by zero. Then the matrix $A$ can be permuted to be a block diagonal matrix. Each block corresponds to a subgroup. Equation (11) demonstrates the principle of $\varepsilon^{-}$ decomposition. A nice property of $\varepsilon$-decomposition is that decomposition results for different thresholds are nested. Namely, for the same matrix A, if $\left|\varepsilon_{1}\right|<\left|\varepsilon_{2}\right|$, the decomposition result for $\varepsilon_{2}$ is always nested in the result for $\varepsilon_{1}$. This property provides the flexibility of choosing series of thresholds $\left|\varepsilon_{1}\right|<\left|\varepsilon_{2}\right|<\ldots<\left|\varepsilon_{N}\right|$ for multi-layered decomposition while not requiring significant increase of computational complexity for introducing more than one threshold.

$$
\left[\begin{array}{lll}
3.0 & 0.4 & 0.2 \\
1.3 & 2.0 & 0.2 \\
0.1 & 0.3 & 5.0
\end{array}\right] \stackrel{\varepsilon=0.5}{\longrightarrow}\left[\begin{array}{llll}
\lceil 3.0 & 0.4\rceil & \\
\lfloor 1.3 & 2.0 & & \\
& & \lceil 5\rceil
\end{array}\right]
$$

\section{Multi-layer Information Structure}

The desired property of an online monitoring framework is that the communication structure be implemented seamlessly under the present hierarchical industry organization. We propose a multi-layered information structure which combines the advantages from both decomposition methods. Fig. 2 shows the conceptual framework of the proposed information structure. Overlapping decomposition is applied to the top layer based on the administrative control areas ${ }^{4}$. At the middle layer, the epsilon decomposition is applied to decompose each control area's Jacobian submatrix into disjointed subgroups.

of the qualitative properties change significantly for the coupled $P-Q$ model. We are currently pursuing research on this.

${ }^{4}$ It is generally more difficult for a control area to gather information outside its administrative boundary. After deregulation, such difficulty seems more significant.
As explained in the previous subsection, the nested property of epsilon decomposition enables series of decomposition levels without too much increase of computational complexity. Our proposed PI is computed at each layer, indicating the severeness of system load level in a bottom-up fashion. The benchmarks for PIs in each subgroup indicating the abnormal load conditions are obtained from offline simulation and training [9].

Each decomposed subgroup contains critical information to monitor the quasi-static voltage conditions in the corresponding area. Higher layer contains more information about the whole system, but is generally more computationally complex. One of the open questions is up to what level of decentralization the quasi-static voltage monitoring can be effective. In the next section, two multicontrol-area examples are used to illustrate our PI under such multi-layered information structure.

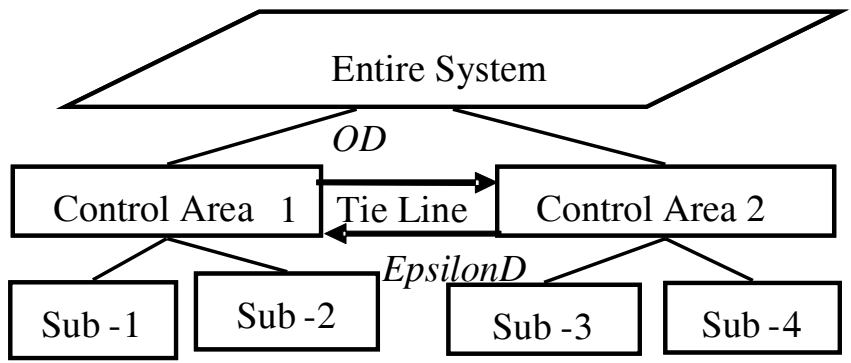

$O D$ : Overlapping decomposition;

EpsilonD : Epsilon decomposition.

Fig. 2. Conceptual framework of a multi-layered information structure.

\section{ILLUSTRATION ON TwO SYSTEMS}

\section{A. 7-Bus System}

Fig. 3 shows a seven-bus system. The dotted line separates three administrative areas. They are connected with each other via tie lines. In the simulation, all the lines are considered to be lossless, and load level is uniformly increased at all buses until quasi-static voltage collapse, i.e. the power flow equations do not have solutions. In this case, none of the generators reached reactive output limit.

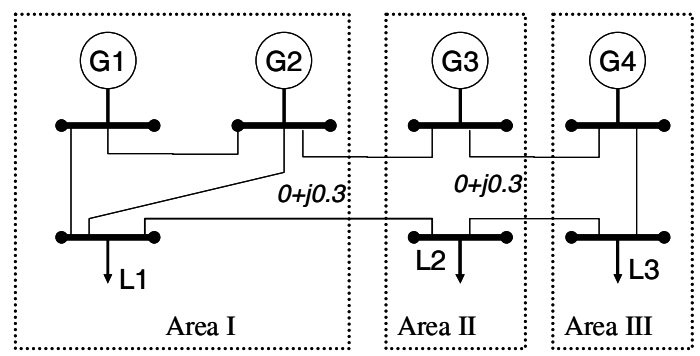

Fig. 3. One line diagram for a three-control-area system.

Fig. 4 demonstrates the minimum singular value (currently frequently used PI) of the system Jacobian matrix, as well as the minimum singular value for block submatrices of $J_{Q V}$ resulted from overlapping decomposition corresponding to three control areas. The following are the observations: (1) 
when the system is close to quasi-static voltage collapse, the minimum singular value of the load flow Jacobian is close to zero. In other words, the currently frequently used PI is effective for centralized scheme; (2) by simply looking at the decomposed submatrices' minimum singular value, one can not tell if the system is close to collapse, namely, the minimum singular value is not an appropriate PI for decentralized monitoring quasi-static voltage problem. However, the "slopes" of the four subplots show qualitatively different values as the system approaches stressed load level. In other words, the proposed PI is capable of detecting stressed system load conditions at control-area level. Table I lists the proposed PI numerical values for the overlapping decomposed control areas' submatrices. For this particular example, control area I, II and III can all use 10.0 as the threshold of the proposed PI to indicate system stressed load level.

Moreover, for each control area's overlapping decomposed submatrices, nested epsilon decomposition is applied. In this example, there is only one load bus corresponding to each control area. So, the next step towards decentralization is at individual bus level. Table II shows the values of proposed PIs at local level for three load buses when the load level gradually increases until collapse. Our proposed PI, although not showing as significant changes as in Table I, still demonstrates qualitatively different values in stressed load level (load level=3.9) compared with normal load level. Therefore, in this particular example, the proposed PI can indicate the stressed load level even at the very bottom layer of the information structure, i.e., at load bus level.

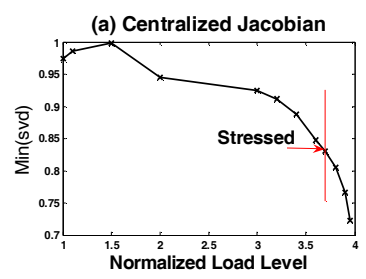

(c) OD for $\mathrm{J}_{\mathrm{QV}}$ in area II

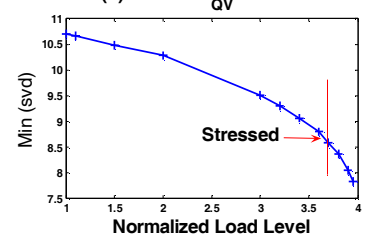

Fig. 4. Minimum singular value of seven-bus-system load Jacobian, and overlapping decomposed $J_{Q V}$ in area I, II and III, respectively.

TABLE I: PROPOSED PIS FOR SYSTEM JACOBIAN AND OVERLAPPING DECOMPOSED QV SUBMATRICES

\begin{tabular}{c|ccccc}
\hline Normalized Load Level $\left(S_{0}\right)$ & 1.0 & 1.5 & 3.0 & 3.6 & 3.9 \\
\hline PI $(J)$ (centralized) & 1.0 & 0.9 & 0.5 & 1.5 & 7.3 \\
PI $(\partial Q / \partial V)$ (area I) & 1.0 & 0.8 & 2.1 & 4.2 & 10.7 \\
PI $(\partial Q / \partial V)$ (area II) & 1.0 & 1.5 & 4.0 & 8.0 & 15.3 \\
PI $(\partial Q / \partial V)$ (area II) & 1.0 & 1.7 & 4.6 & 9.2 & 17.3 \\
\hline
\end{tabular}

TABLE II: PIS FOR EPSILON DECOMPOSED QV SUBMATRICES IN EACH CONTROL AREA

\begin{tabular}{c|ccccc}
\hline Normalized Load Level $\left(S_{0}\right)$ & 1.0 & 1.5 & 3.0 & 3.6 & 3.9 \\
\hline PI $(\partial Q / \partial V)(\mathrm{L} 1$, area I) & 1.0 & 2.0 & 2.0 & 4.0 & 4.0 \\
PI $(\partial Q / \partial V)(\mathrm{L} 2$, area II $)$ & 1.0 & 1.7 & 2.7 & 5.3 & 8.0 \\
PI $(\partial Q / \partial V)(\mathrm{L} 3$, area II) & 1.0 & 2.0 & 3.0 & 4.0 & 6.0 \\
\hline
\end{tabular}

\section{B. IEEE Reliability Test System (73 Buses)}

IEEE Reliability Test System (RTS-1996) is developed primarily for power system reliability evaluation methodology [12]. We use this 73-bus, three-control-area network, as our second test bed. Fig. 5 shows the sketch of RTS-1996 system. In contrast with the system-wide load level increase in the previous example, we focus on the quasi-static voltage problem related with local load increase. Load level at bus \#308 in control area III is increased and the neighboring generator at bus \#307 is balancing the incremental real power until the power flow equation fails to converge.

During this process, two generators' reactive power output limits were reached, indicating certain loss of controllability of these two generators' (Bus\# 307 and \#313) output voltages. Such event is manifested by the increase of dimension by two for the power flow Jacobian matrix.

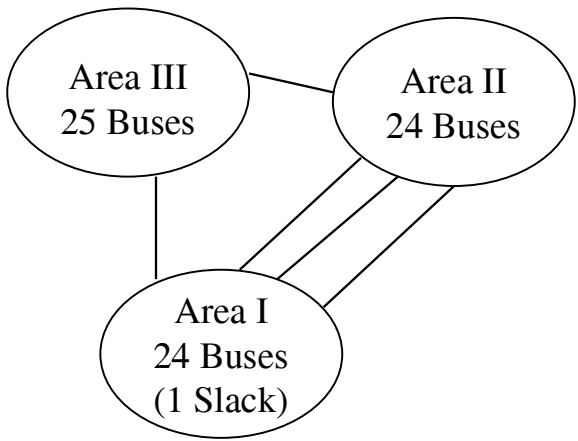

Fig. 5. IEEE RTS-1996 system sketch

Fig. 6 demonstrates the PI currently used at system and control-area levels as the load increases until voltage collapse. For centralized system Jacobian, minimum singular value indicates the proximity of quasi-static limit in an effective way. However, simply computing the value of minimum singular value of submatrix at control area level does not provide much information about system conditions. Our proposed PI, on the other hand, can do so at the control-area level because subplot (d) in Fig. 6 shows qualitative slope change when the system is close to the stressed load conditions. The proposed PI functions effectively at the decentralized control area level. Moreover, the qualitative slope change in (d) coincides with the loss of controllability (sample points 5 and 8).

Besides the overlapping decomposition at control-area level, the authors explore further how effective the proposed "decentralized" PI can be. Nested epsilon decomposition with series of $\varepsilon$ is applied to submatrix of $\mathrm{J}_{\mathrm{QV}}$ corresponding to area III, since this experiment is concerned with a local problem within this area. Fig. 7 below shows the effectiveness of PI at 
different levels of decentralization. From bottom up, three curves correspond to overlapping decomposed $\mathrm{J}_{\mathrm{QV}}$ in area III, largest submatrix resulting from epsilon decomposition of $\mathrm{J}_{\mathrm{QV} \_ \text {ArealII }}$, and local bus $\partial \mathrm{Q} / \partial \mathrm{V}$ at node \#308, respectively. The sharpest slope change corresponds to the control-area layer. The middle curve that results from epsilon decomposition corresponds to 6 nodes around bus \#308. Only requiring the singular value decomposition of $6 * 6$ matrices, the middle curve can still indicate the load severity fairly effectively when the system is stressed. Further decomposed, the top curve shows the numerical value of $\partial \mathrm{Q} / \partial \mathrm{V}$ at bus $\# 308$. Although the top curve slightly changes its slope as the system comes close to collapse, the indication of whether the system is stressed or not becomes more ambiguous. The implication is that the more decentralized the less effective the proposed PI is. There is a tradeoff between the level of decentralization and the effectiveness of the proposed PI.
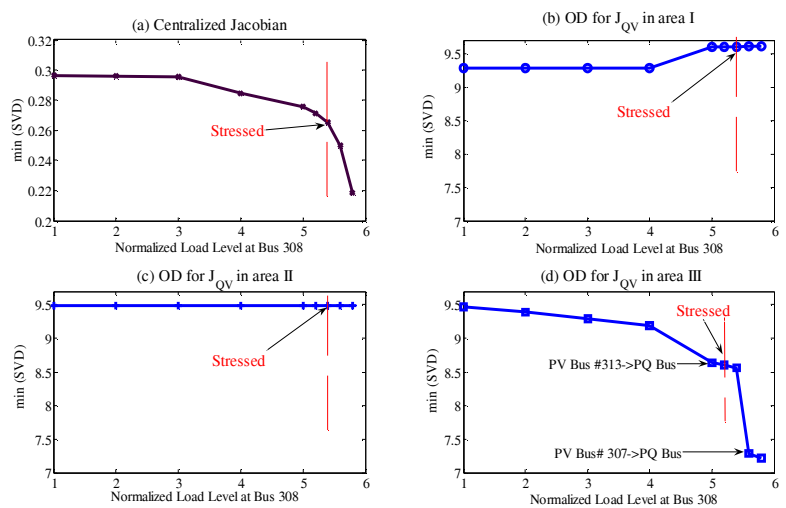

Fig. 6. Minimum singular value of IEEE RTS-1996 system load Jacobian, and overlapping decomposed $J_{Q V}$ in area I, II and III, respectively.

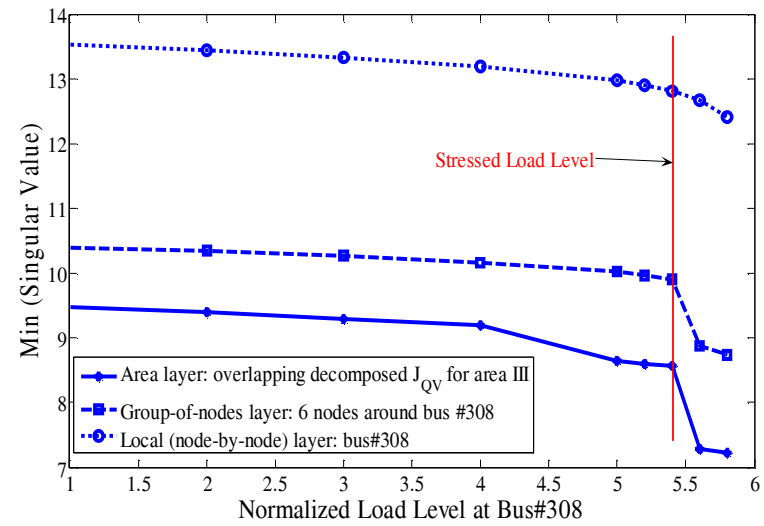

Fig. 7. Effectiveness of PIs at different layers of $J_{Q V}$ decomposition.

\section{Discussion of the control-area decomposition method}

In [13] we explored the two decomposition methods individually. In this paper, we introduce an information flow structure which is the combination of the two decomposition methods. The difference is mainly at the control-area level. It is worthwhile to compare the difference between (1) controlarea-based overlapping decomposition and (2) epsilon decomposition with a choice of $\boldsymbol{\varepsilon}$ such that the result is as close as possible to the control-area-partition.

Table III and IV show the comparison of these two approaches for the 7-bus system and RTS system, respectively. One of the main differences is that the epsilon decomposition is a disjoint partition of a system, while the overlapping decomposition includes tie-line nodes in both neighboring areas' submatrices. The advantage of doing overlapping decomposition at control-area level is that by incorporating the tie-line information, each control area has the critical information about the impact from the neighboring areas.

The performance indices computed from the two decomposition methods at control-area level are also compared. For the 7-bus system, Table I and II are the PIs calculated using overlapping and epsilon decomposition, respectively. Both PIs can qualitatively estimate the severeness of the load level, while the PIs from the overlapping decomposition change more significantly. For the RTS-1996 system, Table V shows the PIs for area III when we apply control-area overlapping and epsilon decomposition. Both PIs have similar numerical values when the system is close to the quasi-static voltage stability limit (when $S_{0}=5.2$ ). However, the control-area overlapping decomposition indicates a qualitative different value when $S_{0}=4.0$, which is due to the event that PV bus \#313 reaches the reactive power output limit. Such event is not observed by the PI resulted from epsilon decomposition.

TABLE III

CONTROL-AREA LAYER DECOMPOSITION FOR 7-BUS SYSTEM

\begin{tabular}{c|c}
\hline $\begin{array}{c}\text { Overlapping Decomposition for } \\
J_{Q V}\end{array}$ & $\begin{array}{c}\text { Epsilon Decomposition for } \\
J_{Q V}\end{array}$ \\
\hline$\{\mathrm{L} 1, \mathrm{~L} 2\}$ & $\{\mathrm{L} 1\}$ \\
\hline$\{\mathrm{L} 1, \mathrm{~L} 2, \mathrm{~L} 3\}$ & $\{\mathrm{L} 2\}$ \\
\hline$\{\mathrm{L} 2, \mathrm{~L} 3\}$ & $\{\mathrm{L} 3\}$ \\
\hline
\end{tabular}

TABLE IV

CONTROL-AREA LAYER DECOMPOSITION FOR RTS SYSTEM

\begin{tabular}{c|c}
\hline Overlapping Decomposition for $J_{Q V}$ & $\begin{array}{c}\text { Epsilon Decomposition for } \\
J_{Q V}\end{array}$ \\
\hline$\{101,102,107,114,115,116,118$, & $\{101,102,107,114,115$, \\
$119,121,122,123,215,325\}$ & $116,118,119,215\}$ \\
& $\{122,123\}$ \\
\hline$\{201,202,207,213,214,215,216$, & $\{201,202,207,213,214$, \\
$218,221,222,223,107,123,318\}$ & $216,218,223\}$ \\
& $\{221,222\}$ \\
\hline$\{301,302,307,313,314,315,316$, & $\{301,302,307,313,314$, \\
$318,321,322,323,121,223\}$ & $315,316\}$ \\
& $\{318,321\}$ \\
\hline
\end{tabular}

TABLE V

PERFORMANCE INDEX FOR RTS SYSTEM IN CONTROL-AREA LAYER Normalized Load Level $\left(S_{0}\right) \quad\left[\begin{array}{llllll}\hline .0 & 2.0 & 3.0 & 4.0 & 5.0 & 5.2\end{array}\right.$

\begin{tabular}{l|cccccc} 
PI_OD (area III) & 1.0 & 1.3 & 1.3 & 7.1 & 2.2 & 81.5 \\
PI_ED (area III) & 1.0 & 1.2 & 1.6 & 2.5 & 5.0 & 82.7 \\
& & & & & &
\end{tabular}




\section{CONCLUSIONS}

A novel performance index is proposed to show the potential of online monitoring of quasi-static voltage problem in a decentralized way. Such performance index, which is based on the sensitivity of minimum singular value of the load flow Jacobian matrix with respect to the corresponding system load level, can qualitatively estimate the closeness to the quasi-static voltage stability limit of an interconnected power system. A multi-layered information structure, which combines the control-area based overlapping decomposition and nested epsilon decomposition, is introduced to specify the computation of PI at different levels of system decomposition. At control-area layer, overlapping decomposition is used to decompose the interconnection, requiring only tie-line information exchange among administrative control areas. At lower layers, the epsilon decomposition is used. Such combination takes the "administrative barrier" into consideration while offering a flexible level of decentralization. Therefore, it can be seamlessly incorporated in today's horizontally partitioned industry structure.

Two multi-control-area systems are used to illustrate the proposed performance index and the multi-layered information structure. The conclusions are that our proposed PI detects the quasi-static load severeness in a decentralized way, both for system-wide and local problems. The PI is less effective at lower layers, which corresponds to more decentralized levels. However, it is computationally more difficult to obtain the PI at higher layers. Such observation reveals the tradeoff between the level of decentralization and the effectiveness of the PI for indicating potential quasi-static voltage problems.

However, the question of how to analytically decide the level of aggregation/decomposition to guarantee the performance of such proposed index still remains open. Another aspect of further research is to investigate the general applicability of the proposed PI.

\section{ACKNOWLEDGEMENT}

The authors acknowledge the comments made by Prof. Gerald Hedyt from Arizona State University at the 2006 North American Power Symposium with respect to singular value versus eigenvalue approach. The first author greatly acknowledges the discussion with Prof. Bruce Krogh in ECE Department at Carnegie Mellon University. The first author also acknowledges the discussion with Huaiwei Liao and Bo $\mathrm{Li}$, both of whom are PhD students in ECE Department at Carnegie Mellon University.

\section{REFERENCES}

[1] M.D. Ilić, E.H. Allen, J.W. Chapman, C.A. King, J.H. Lang, and E. Litvinov, "Preventing future blackouts by means of enhanced electric power systems control: from complexity to order," Proceedings of the IEEE, vol. 93, pp.1920-1941, Nov. 2005.

[2] J.W. Chapman, M.D. Ilić, C.A. King, L.Eng, and H. Kaufman, "Stabilizing a multi-machine power system via decentralized feedback linearizing excitation control," IEEE Trans. Power Systems, vol. 8, pp. 830-839, Aug. 1993.
[3] A. Thatte, and M.D. Ilić, "An assessment of reactive power/voltage control devices in distribution networks," in Proc. IEEE Power Engineering Society 2006 General Meeting, Paper \# 06GM1233, June 2006, Montreal, Canada.

[4] T.Van Cutsem and R. Mailhot, "Validation of a fast voltage stability analysis method on the Hydro-Quebec system," IEEE Trans. Power Systems, vol. 12, pp. 282-292, Feb. 1997.

[5] M. D. Ilić and J. Zaborszky, Dynamics and Control of Large Electric Power Systems, New York: Wiley Interscience, 2000, pp. 405-450.

[6] P. Kundur, Power System Stability and Control, McGraw-Hill, 1994, pp. 960-1019.

[7] P.W. Sauer and M.A. Pai, "Power system steady-state stability and the load-flow Jacobian," IEEE Trans. Power Systems, vol. 5, pp. 1374-1383, Nov. 1990.

[8] P-A Lof, G. Andersson, and D.J. Hill, "Voltage stability indices for stressed power systems," IEEE Trans. Power Systems, Vol. 8, No. 1, pp.326-335, February 1993.

[9] J. Ilić, L. Xie, and M.D. Ilić, "Critical voltage monitoring using sensitivity and optimal information machine", Proceedings of North American Power Symposium, 2006.

[10] M.E. Sezer and D.D. Siljak, "Nested epsilon decompositions of linear systems: weakly coupled and overlapping blocks," SIAM Matrix Analysis Application, Vol. 12, No. 3, pp.521-533, July 1991.

[11] M.D. Ilić, "Reconciling hierarchical control and open access in the changing electric power industry," Proceedings of IEEE Special Issue on Modeling, Identification, and Control of Large-scale Dynamical Systems, 2007 (Invited paper).

[12] J.M.S. Pinheiro, C.R.R. Dornellas, M.Th. Schilling, A.C.G. Melo, and J.C.O. Mello, "Probing the new IEEE reliability test system (RTS-1996): HL-II assessment”, IEEE Trans. Power Systems, Vol. 13, No.1, pp. 171176, Feb. 1998.

[13] L. Xie, J. Ilić, and M.D. Ilić, "Towards grid modernization through enhanced communications and computing: novel performance index and information structure for monitoring voltage problems", Proceedings of North American Power Symposium, 2006.

\section{BIOGRAPHIES}

Le Xie (S'05) was born in 1983 in Ganzhou, China. He received his B.E. in Electrical Engineering in 2004 from Tsinghua University, Beijing, China. Afterwards he came to the USA for graduate study. He got his master's degree in Engineering Sciences from Harvard University in June 2005. Currently he is pursuing $\mathrm{PhD}$ in the Department of Electrical and Computer Engineering at Carnegie Mellon University.

His industry experience included an internship (Jun 2006-Aug 2006) at Market Monitoring Department, ISO-New England. His research interest includes power system control and electricity market.

Jovan Ilić (M'06) received his B.S from The University of Illinois, ChampaignUrbana in 1989 and his M.S. and Ph.D. in 1992 and 2001 respectively from The University of Tennessee, Knoxville. His interests are in advanced, adaptive dynamic systems, and secure information technology as applied in digital control systems. He spent a few years in computer industry as a full time employee and independent consultant. Currently, he is a researcher in the area of deregulated power systems at the Carnegie Mellon University.

Marija D. Ilić (M'80-SM'86-F'99) was an assistant professor at Cornell University, Ithaca, NY, and tenured Associate Professor at the University of Illinois at Urbana-Champaign. She was then a Senior Research Scientist in Department of Electrical Engineering and Computer Science, Massachusetts Institute of Technology, Cambridge, from 1987 to 2002. She is currently a Professor at Carnegie Mellon University, Pittsburgh, PA, with a joint appointment in the Electrical \& Computer Engineering and Engineering \& Public Policy Departments.

She has 30 years of experience in teaching and research in the area of electrical power system modeling and control. Her main interest is in the systems aspects of operations, planning, and economics of the electric power industry. She has co-authored several books in her field of interest.

Prof. Ilić is an IEEE Distinguished Lecturer. 